\title{
Seeing red? Putting sportswear in context
}

\author{
Arising from: R. A. Hill \& R. A. Barton Nature 435, 293 (2005)
}

The shirt colour worn by sportsmen can affect the behaviour of the competitors ${ }^{12}$, but Hill and Barton ${ }^{3}$ show that it may also influence the outcome of contests. By analysing the results of men's combat sports from the Athens 2004 Olympics, they found that more matches were won by fighters wearing red outfits than by those wearing blue; they suggest that red might confer success because it is a sign of dominance in many animal species and could signal aggression in human contests. Here we use another data set from the 2004 Olympics to show that similar winning biases occur in contests in which neither contestant wears red, indicating that a different mechanism may be responsible for these effects.

If, as Hill and Barton claim, there is something special about the colour red, then contests using other colour pairings should not be biased. We tested whether this could be the case by analysing data (www.athens2004.com) from contests in which red was not used for competitors' outfits: in judo matches, one player wears blue and the other wears white. We followed the methodology originally used by Hill and Barton ${ }^{3}$ for boxing, tae kwon do, and Greco-Roman and freestyle wrestling.

After ensuring that outfit (judogi) colour in judo contests was allocated at random (www.ijf.org), we found a significant winning bias for players wearing blue compared with those wearing white $\left(\chi^{2}=7.34, \mathrm{~d} . \mathrm{f}=1\right.$, $P<0.01$ ), and a similar effect of contest symmetry on winning bias to that reported by Hill and Barton (Fig. 1). We also found the winning bias for players wearing blue when considering only contests in the first round of competition $\left(\chi^{2}=4.85, \mathrm{d.f}=1, P<0.05\right)$. This result excludes the possibility that the observed bias might arise through skilled contestants being placed, by chance, in draw positions where they wear blue more often as they progress through the competition.

Our results indicate that there is nothing

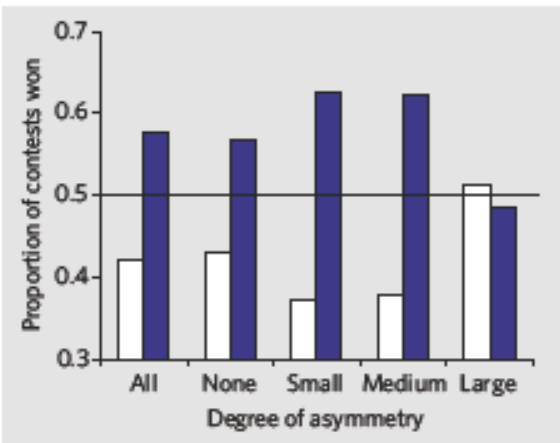

Figure 1 | Influence of judogicolour on the outcome of judomatches in the Athens 2004 Olympics. The black line at 0.5 indicates the expected proportion of wins by blue or white under the null hypothesis that colour has no effect on contest outcome. Blue bars, proportion won by players in blue; white bars, proportion won by players in white. There were significant differences between the number of blue and white wins for all contests combined $\left(\chi^{2}=7.34\right.$, d.f. $=1, P=0.007$ ). This difference is most evident at low degrees of asymmetry in relative ability of the two competitors in each bout (subdivided using quartiles of points difference, after Hill and Barton ${ }^{3}$ ). Differences between the number of white and blue wins were as follows: no asymmetry $\left(\chi^{2}=1.53\right.$, d.f. $\left.=1, P=0.22\right)$, small asymmetry $\left(\chi^{2}=6.31\right.$, d.f. $\left.=1, P=0.012\right)$, medium asymmetry $\left(\chi^{2}=3.19\right.$, d.f. $\left.=1, P=0.07\right)$ and large asymmetry $\left(\chi^{2}=0.57\right.$, d.f. $=1$, $P=0.81$ ). Number of contests recorded are 301 , $79,99,53$ and 70 , respectively.

inherently special about red in terms of colour-associated winning biases.

We can think of no plausible evolutionary explanation based on animal behaviour or evolutionary psychology that might account for a winning bias for blue contestants. We propose instead that outfit colour affects opponent visibility, which is crucial for avoidance and interception, and for anticipating behaviour. Visual abilities that could influence sporting performance include being able to follow rapidly moving objects and perform fast visual searches ${ }^{4-6}$. And the hue, saturation, brightness and contrast of an object (or opponent) could enable it to be picked out against its background ${ }^{7, s}$. These factors are critical for combat sports and for detecting teammates on the field of play (http://wwwwliv.ac.uk/research intelligence/issue1/manunit.html; 1999).

In judo, the white judogi is likely to be perceived as brighter than the blue and may have higher contrast against the background. Men wearing blue may therefore have a visual advantage in being able to anticipate their (white) opponents' moves. We do not know the reflectance spectra, lighting arrangements or other visual factors that might have affected the visual salience of the red and blue outfits worn in the sports studied by Hill and Barton. Although our hypothesis is untested, visibility differences could also explain the biases they found. The visual attributes of sporting wear should therefore be considered in this wider perceptual context.

Candy Rowe ${ }^{\star}$, Julie M. Harrist, S. Craig Roberts; *School of Biology \& Psychology, University of Newcastle, Newcastle upon Tyne NE2 4HH, UK e-mait candy.rowe@nclac.uk

†School of Psychology, University of St Andrews, StMary's College, St Andrews KY16 9JP, UK †Evolutionary Psychology \& Behavioural Ecology Group, School of Biological Sciences, University of Liverpool, Liverpool L69 7ZB, UK

1. Frank, M.G \& Gilovich, T.J.Pers Soc. Ayychal. 54, 74-85 (1988).

2. Mills, B. D.\& French, L. M. J.Hum. Mov. Stud 31,47-60 (1996)

3. Hill, R.A.\& Barton, R. A. Nature 435, 293(2005).

4. Williams, A.M. J.SpartSci. 18,737-750 (2000)

5. Farrow, D. \& Southgate D. Clin Exp Optom. 83, 226-231 (2000)

6. Williams, M. Pychalogist 15,416-417(2002)

7. McKeefry, D. I et al. Invest. Ophthal. Vis Sci. 44, 2267-2276 (200B)

8. Regan, B. C etal. Phil. Trans R. Soc. Lond B356,229-283 (200i).

doi:101038/nature04306

\section{SPORTING CONTESTS}

\section{Hill \& Barton reply}

Replying to: C. Rowe, J. M. Harris and S.C. Roberts Nature 437, doi:10.1038/nature04306 (2005)

Rowe et al. corroborate our finding that the colour of clothing influences the outcome of sporting contests ${ }^{1}$, but they offer a different mechanism to explain the effect. We found that in four combat sports wearing red was consistently associated with improved perfor- mance relative to wearing blue ${ }^{2}$, and argued that wearing red enhances performance through psychological effects on the wearer and/or on the opponent. We suggested that these psychological effects reflect the evolutionary and cultural associations of red with dominance and aggression. Rowe et al. find that, in a fifth combat sport, wearers of blue outperformed wearers of white. They attribute their and our results to perceptual rather than to psychological effects, arguing that visibility of the opponent is the critical factor.

In our view, this visibility explanation is unlikely in a situation where contestants fight at close quarters in brightly lit arenas, as in these combat sports. Crucially, in the combat sports we analysed, the hypothesis of Rowe et al. requires that blue-wearing opponents be more visible than their red-wearing opponents, 